Aim of the study: There is little known about any change in postural balance caused by asymmetrical volume increase due to unilateral upper extremity lymphedema in patients who underwent breast surgery. The aim of this study was to determine whether there is a change in postural balance by measuring postural sway velocity (PSV), center of gravity (CoG) displacement and directional control (DCL) in patients with unilateral upper extremity lymphedema in breast cancer survivors.

Material and methods: Eighteen females 38-60 ( $M=53)$ years old diagnosed with upper extremity lymphedema due to breast cancer surgery, and 18 healthy females with similar ages $(M=52.5)$ were assessed using the Balance Master system (Neuro Com, Clackamas, USA). Unilateral stance (US) and bilateral stance (BS) tests in eyes open and closed conditions and the limit of stability (LOS) test were applied to quantify postural sway velocity (PSV), CoG displacement, and directional control (DCL).

Results: The lymphedema group showed a significant increase in PSV in the US test on the ipsilateral leg with eyes open $(p=0.02)$ and eyes closed $(p=0.005)$ as well as on the contralateral leg with eyes open $(p=0.004)$ and eyes closed $(p=0.0001)$. Average displacement and position of the CoG were $25 \%$ of LOS $(p=0.0001)$ towards the lymphedema side and 60.6 degrees respectively. DCL in the lymphedema group was significantly lower in forward $(p=0.0001)$, back $(p=0.003)$, ipsilateral $(p=0.002)$, and contralateral $(p=0.03)$ directions.

Conclusions: These findings suggest that unilateral upper extremity lymphedema may have challenging effects on postural balance.

Key words: upper extremity, lymphedema, postural stability, postural sway, breast cancer.

Contemp Oncol (Pozn) 2014; 18 (4): 279-284 DOI: $105114 /$ wo.2014.44120

\section{Unilateral upper extremity lymphedema deteriorates the postural stability in breast cancer survivors}

\author{
Salih Angin, Didem Karadibak, Tuğba Yavuzşen, ilkşan Demirbüken
}

Dokuz Eylül University, School of Physical Therapy and Rehabilitation, Sağlık Kampusü, Izmir, Turkey

\section{Introduction}

Any deviation in the location of the centre of gravity (CoG) by leaning towards any direction on the supporting surface is described as postural sway, and can be assessed by using a force platform. The cause of sway may be described as an active search process to find the best possible position at a given moment, and an output of a control process to maintain this position. However, many factors may play a certain role in degradation of the postural stability by means of increasing postural sway such as disability of the sensory-motor system [1] as well as weight asymmetry such as amputation [2] and unilateral weight carriage [3] or unilateral volume change in the upper body [4].

Lymphedema is a severe, chronic and progressive complication in breast cancer survivors; it causes chronic and progressive swelling of the arm, shoulder, neck, or upper trunk from physical compression or disruption of the axillary lymphatic channels from surgery or radiation therapy [5]. Untreated lymphedema may also cause chronic inflammation, cellulitis, pain, fatigue, inability to work, cosmetic deformity, and a significant decrease in mobility and functional status, and use of the affected extremity [6-8]. Sensations of heaviness, fullness, tightness and paresthesia in the affected side may disturb upright posture [9]. Diminished cutaneous sensation and limitation in fine balancing movement of the upper extremity, and probably an increased limb volume compared to the normal arm, may reduce the ability to maintain postural stability [10]. Until now, not enough attention has been paid to investigate the potential effect of unilateral volume increase caused by upper extremity lymphedema on postural stability. According to an earlier study, lymphedema did not cause an alteration of spinal posture [11]. It was the only study that currently exists in the literature investigating the relationship between lymphedema and spinal posture, but it was not clear whether any change was found in overall postural stability and position of the CoG. To date, there have been no published data known to the present authors whether the CoG is displaced because of asymmetrical volume increase. Therefore, the aim of this study was to determine whether there is a change in overall postural stability by measuring postural sway velocity, displacement of CoG and directional control in patients with unilateral upper extremity lymphedema.

\section{Material and methods}

\section{Participants}

Eighteen females patients aged 38-60 years old diagnosed with upper extremity lymphedema due to breast cancer surgery were included in the study. The participants had modified radical mastectomy. Axillary lymph node dissection was performed to remove levels I and II of underarm lymph nodes. 
Average duration of lymph node removal was $15.2 \pm 9.5$ (5-25) years, and average duration of the lymphedema was $3.3 \pm 3.5$ (1-7) years. All participants had moderate lymphedema according to the lymphedema severity assessment described elsewhere [12]. Eligible participants were required to have completed at least one year after surgery and adjuvant therapy [13]. Adjuvant radiation was delivered to the affected breast (usually the anterior chest wall) and to the lymph nodes under the arm at the collarbone.

Eighteen healthy females with similar ages were recruited for the control group. Demographic data for both groups are presented in Table 1. Subjects with severe arthritis or orthopedic conditions in their lower extremity, or poor conditioning, acute pain in any reason, neurological disorders including visual problems, and patients older than 65 years old were excluded from the study. All participants signed an informed consent form. The study was approved with code $860-\mathrm{GOA}$ by the University Ethical Committee and all tests were conducted according to the latest revision of the Declaration of Helsinki.

\section{Equipment}

All the participants were assessed using the Balance Master system (version 8.1, NeuroCom, International, Clackamas, Oregon, USA). The Balance Master system is a computerized device that provides real-time movement analysis on a force platform for objective assessment and comprehensive documentation of postural control. It has the capacity to objectively assess the individual's ability to perform specific balance tasks essential to daily living [14]. The Balance Master consists of a force platform connected to a computer, with a software program that continuously monitors the position and movement of the center of gravity $(\mathrm{CoG})$. The patient stands on the force platform and faces the monitor. Force sensors under the force platform measure the vertical forces exerted by the patient's feet. The computer receives force measurements from the dual force platform, analyses the information, generates a screen display, and prints a graphical and numerical report. The data and results from each test are stored on the hard disk of the computer in a uniquely named file [14, 15].

\section{Test procedure}

Unilateral stance (US) test, bilateral stance (BS) test and limit of stability (LOS) test were selected for assessment. Three trials were recorded for each test and the average values of the three trials were included in the statis- tical analysis. The length of each trial was 10 seconds for US and BS tests, and eight seconds for the LOS target trial. The subjects were instructed not to consume any caffeine or alcohol for 48 hours prior to the testing procedure. Before starting the test, the subjects were instructed about the test procedure. The subjects were advised to stand as close as possible to a vertical position that they perceived. All tests were performed as described elsewhere [14, 15]:

Unilateral Stance (US) Test: The unilateral stance (US) test was selected to quantify postural sway. Postural sway was assessed by measuring sway velocity of the CoG expressed in degrees per second (Fig. 1). Relative absent of sway was considered as stability. The participants in the study group were asked to stand on their foot on the lymphedema side (ipsilateral foot) over the force platform with eyes open and then with eyes closed as described [14, 15]. If the subjects lost their balance within 10 seconds, the trial was marked as a fall. The same assessments were also performed for the contralateral foot. The US test was performed for the control group while they were standing on their right, and then left foot.

Limits of Stability (LOS) Test: In the present study, the limit of stability (LOS) test was employed to quantify directional control $(\mathrm{DCL})$ within the maximum distance a person can intentionally displace their CoG by leaning their body toward targets on the front, right front, right, right back, back, left back, left and left front directions without losing balance. Scores from front, right front and left front are combined to provide an average forward score. Average backward, right and left scores were also computed from corresponding directions by the system software (NeuroCom Data Analyzer 8.1). DCL was quantified as comparing the amount of movement in the intended direction (straight line) toward the target and the amount of extraneous movement away from the straight line [14, 15]. A straight line from the center to the target represents perfect DCL equal to $100 \%$ LOS, while extraneous movements indicate lower DCL and increased postural sway around the straight line (Fig. 2). The subjects were asked to stand on both their feet over the force platform. At the beginning of the test, the position of the CoG was displayed as a cursor at the center of the screen, and the subject could control it by weight shifting. When the sign appeared on one of the eight boxes, the subject leaned quickly and accurately to move the cursor toward the highlighted box and held the cursor in the box until the sign disappeared. The same procedure was followed for the other seven target boxes on the screen.

Table 1. Demographic data for lymphedema and control groups

\begin{tabular}{|c|c|c|c|c|c|c|}
\hline \multirow{2}{*}{$\begin{array}{l}\text { Demographic } \\
\text { variables }\end{array}$} & \multicolumn{2}{|c|}{ Lymphedema group } & \multicolumn{2}{|c|}{ Control group } & \multirow[t]{2}{*}{ z } & \multirow[t]{2}{*}{$p$} \\
\hline & M & $(95 \% \mathrm{Cl})$ & M & $(95 \% \mathrm{Cl})$ & & \\
\hline Age (years) & 53.0 & $(50.0-56.6)$ & 52.5 & $(46.4-60.2)$ & 0.19 & 0.85 \\
\hline Body weight (kg) & 75.0 & $(66.8-87.8)$ & 68.5 & $(62.8-74.6)$ & 2.01 & 0.05 \\
\hline Body height $(\mathrm{cm})$ & 161.0 & (159.0-167.6) & 164.0 & $(160.0-166.8)$ & 0.20 & 0.84 \\
\hline Body mass index & 29.7 & $(24.9-32.4)$ & 25.7 & $(23.8-27.8)$ & 1.84 & 0.07 \\
\hline
\end{tabular}

$\mathrm{M}$-median; $\mathrm{Cl}$-confidence interval 


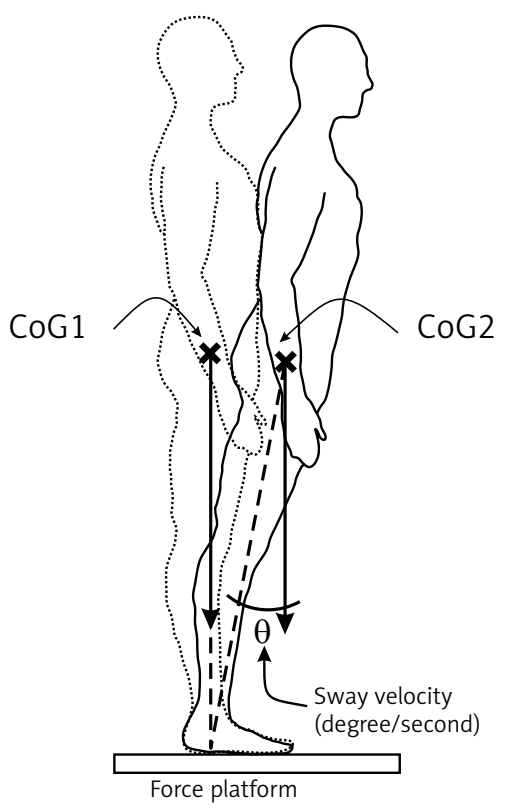

Fig. 1. The unilateral stance test quantifies postural sway velocity in a patient standing on either the right or left foot on the force platform with eyes open and eyes closed. CoG1 represents center of gravity over the support base at the beginning of the unilateral stance test. At a given moment, center of gravity is displaced to CoG2 position due to postural sway. Sway velocity is described as swapped degrees $(\theta)$ per second during movement of CoG from first to second position

Bilateral Stance (BS) Test: The bilateral stance test was selected to quantify the position of the CoG in the upright position with eyes open and eyes closed. The subjects were asked to stand on both their feet over the force platform and hold their position as still as possible to minimize the postural sway. CoG alignment reflects the patient's CoG position relative to the center of the support base. Data gathered from eyes open and eyes closed conditions were computed by the system software for average CoG displacement. Amount of displacement was represented by the distance between the center of the support base and the point that the CoG displaced, and expressed as the percentage of the LOS. The position of the CoG was exhibited by a degree value in one of the four quadrants in the elliptic shape representing the LOS (Fig. 3).

\section{Statistical analysis}

The Mann-Whitney $U$ test was performed to compute the difference between groups using the SPSS 11 package for Windows. This nonparametric test was selected because of the small sample size and lack of normal distribution of data in each group. As there was no significant difference between data obtained from right and left sides of the participants in the control group, data collected from the right side were included in the statistical analysis. Statistical significance was set at $\alpha<0.05$ for all analyses.

\section{Results}

The results revealed that unilateral upper extremity lymphedema had a significant effect on postural stabili-

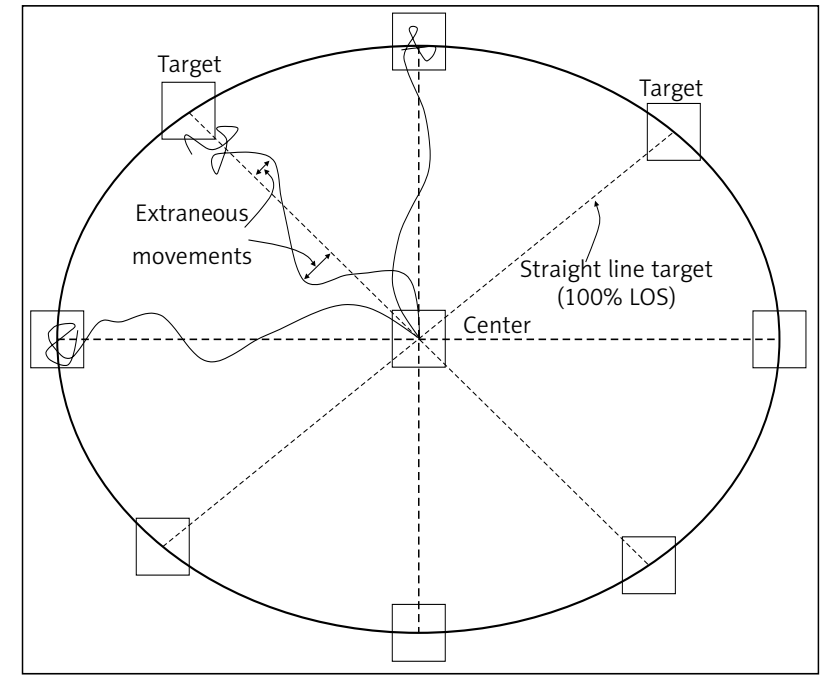

Fig. 2. Limit of stability test is employed to quantify directional control within the maximum distance a person can intentionally displace their center of gravity by leaning their body toward a given direction. Directional control is a comparison of the amount of movement in the intended direction (towards the target) to the amount of extraneous movement while leaning towards the target. Straight line from center to target represents perfect directional control equal to $100 \%$ of limit of stability, while extraneous movement indicates postural sway around the straight line

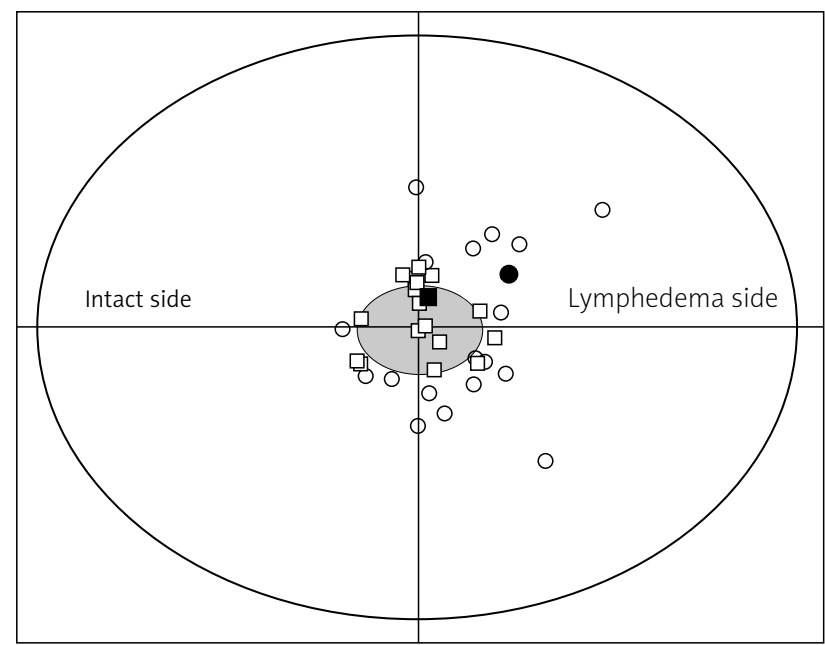

Fig. 3. Circles represent center of gravity of each participant and filled circle indicates median for center of gravity position in lymphedema group. Squares represent individual center of gravity, and filled square indicates median for center of gravity position in control group. Center of gravity position was 60.65 degrees at first quadrant of ellipse shape boundary of limit of stability. Displacement of center of gravity from center of the support base was 25\% of limit of stability in lymphedema group

ty. During the US, postural sway velocity on the ipsilateral foot with eyes opened and eyes closed, and on the contralateral foot with eyes opened and eyes closed were found to be higher in the lymphedema group than the control group (Table 2). Difference of the sway velocity between the two groups were significant for the ipsilateral leg with 
Table 2. Comparison of postural stability variables for lymphedema and control groups

\begin{tabular}{|c|c|c|c|c|c|c|}
\hline \multirow[t]{2}{*}{ Variables } & \multicolumn{2}{|c|}{ Lymphedema group } & \multicolumn{2}{|c|}{ Control group } & \multirow[t]{2}{*}{ Z } & \multirow[t]{2}{*}{$\mathrm{p}$} \\
\hline & M & $(95 \% \mathrm{Cl})$ & M & $(95 \% \mathrm{Cl})$ & & \\
\hline \multicolumn{7}{|l|}{ US test for PSV (degrees/second) } \\
\hline EO ipsilateral side vs. control & 1.62 & $(1.02-4.60)$ & 0.90 & $(0.84-1.22)$ & 2.30 & 0.02 \\
\hline EO contralateral side vs. control & 2.35 & $(0.90-4.70)$ & 0.90 & $(0.84-1.22)$ & 2.87 & 0.004 \\
\hline EO ipsilateral side vs. contralateral side & 1.62 & $(1.02-4.60)$ & 2.35 & $(0.90-4.70)$ & 0.73 & $0.47 \mathrm{NS}$ \\
\hline EC ipsilateral side vs. control & 3.97 & $(2.64-4.64)$ & 1.90 & $(1.54-3.02)$ & 2.80 & 0.005 \\
\hline EC contralateral side vs. control & 5.37 & $(3.52-8.46)$ & 1.90 & $(1.54-3.02)$ & 3.88 & 0.0001 \\
\hline EC ipsilateral side vs. contralateral side & 3.97 & $(2.64-4.64)$ & 5.37 & $(3.52-8.46)$ & 2.12 & 0.03 \\
\hline \multicolumn{7}{|l|}{ LOS test for DCL (\% LOS) } \\
\hline Forward lymphedema group vs. control & 74.5 & $(71.4-81.0)$ & 86.5 & $(84.4-89.0)$ & 4.12 & 0.0001 \\
\hline Backward lymphedema group vs. control & 72.0 & $(58.8-77.2)$ & 80.0 & $(76.8-84.6)$ & 2.98 & 0.003 \\
\hline Ipsilateral side vs. control & 75.5 & $(69.0-80.6)$ & 84.0 & $(80.0-86.6)$ & 3.04 & 0.002 \\
\hline Contralateral side vs. control & 84.0 & $(80.0-86.6)$ & 79.5 & $(77.4-83.6)$ & 2.12 & 0.034 \\
\hline Ipsilateral side vs. contralateral side & 75.5 & $(69.0-80.6)$ & 79.5 & $(77.4-83.6)$ & 1.33 & $0.18 \mathrm{NS}$ \\
\hline \multicolumn{7}{|l|}{ BS test for CoGD (\% LOS) } \\
\hline Lymphedema group vs. control & 25.0 & $(22.0-34.8)$ & 15.0 & $(13.4-18.0)$ & 4.64 & 0.0001 \\
\hline
\end{tabular}

NS - non-significant; M - median; CI - confidence interval; US - unilateral stance; PSV - postural sway velocity; EO - eyes open; EC - eyes closed; LOS - limit of stability; $D C L$ - directional control; BS - bilateral stance; CoGD - center of gravity displacement

eyes open $(Z=2.30, p=0.02)$ and eyes closed $(Z=2.80$, $p=0.005)$, and on the contralateral leg with eyes open $(Z=2.87, p=0.004)$ and eyes closed $(Z=3.88, p=0.0001)$. These results confirm that participants with lymphedema have increased postural sway, indicating poor postural stability compared to the control group. An increased range of postural sway was also detected with the US on the contralateral side in eyes closed conditions when compared to the ipsilateral side $(Z=3.97, p=0.03)$.

Significant displacement of the CoG was found in the lymphedema group towards the ipsilateral side during the BS test, while control group values were within normal limits. The average CoG position was falling in the first quadrant of the ellipse shape boundary of the LOS that was the side of the upper extremity with lymphedema (Fig. 3). Average displacement and position of the CoG were $25 \%$ of $\operatorname{LOS}(Z=4.64, p=0.0001)$, and 60.6 degrees $(Z=2.86, p=0.004)$ respectively (Table 2$)$.

DCL were calculated as the percentage of the total LOS. There was increased extraneous movement but decreased direct movement toward each target in the lymphedema group. DCL values exerted from the lymphedema group were significantly smaller in forward $(Z=4.12, p=0.0001)$, back $(Z=2.98, p=0.003)$, ipsilateral $(Z=3.04, p=0.002)$, and contralateral $(Z=2.12, p=0.03$ ) directions (Table 2$)$. These results also indicate poor directional control in subjects with lymphedema during movement from the center to the targets.

\section{Discussion}

Postural stability is one of the most important components for normal function, and it is necessary in daily activities such as walking or climbing the stairs. The maintenance of postural stability that reflects the contribution of many different joints and muscles manifests as postural sway. While lymphedema is a common health problem of women who suffer from breast cancer that affects their functional status negatively $[7,9,16,17]$, it was not clear that whether there were any changes in postural stability by means of CoG displacement with respect to the center of the support base and postural sway, and directional control in unilateral upper extremity lymphedema. In the present study, the results indicated that there was significant displacement of the CoG towards the lymphedema side and increased postural sway in the lymphedema group.

While the BS requires one to keep the CoG over the center of the support base, asymmetric loading of the extremities results in CoG displacement $[2,18,19]$. A similar result was also found in the present study indicating that the average CoG was displaced towards the lymphedema side at an amount of $25 \%$ of LOS with 60.6 degrees in the first quadrant of the ellipse shape boundary of the LOS (Fig. 3). This clearly indicated the positional change of the CoG towards the lymphedema side due to increased volume and therefore a heavier upper extremity with lymphedema.

Unilateral weight carrying in the upright standing position may be the best example that simulates this condition in the present study to understand the underlying mechanism of why the COG is displaced toward the affected side. Hence, several experimental studies, which were carried out to investigate the effect of unilateral weight carrying on upright posture, revealed that when individuals carry the load on one side, they shift their body weight toward the load and the CoG is displaced to the ipsilateral side of the load $[19,20]$. Besides that, the magnitude of postural sway was found to be affected by unequal body weight distribution [21] as the load displaced the CoG closer to the boundary of the LOS, resulting in decreased postural 
stability. This supports the result in the present study that there was a significant increase in postural sway in the lymphedema group specifically in eyes closed conditions during the US test. The postural sway was even higher on the contralateral side in eyes closed conditions when compared to the ipsilateral side. These findings may indicate that controlling the location of the CoG became difficult because the distance from the local CoG of the upper extremity with lymphedema to the contralateral supporting lower extremity was greater than the distance to the ipsilateral side. A similar result was also obtained by Haddad et al. [19], who found that postural sway was greater on the contralateral side with unilateral weight carrying.

Similar to the findings in the current study and the unilateral weight carrying studies in the literature, Greitemann et al. [2] found that unilateral upper limb amputation also caused CoG displacement toward the normal side and therefore resulted in the development of an asymmetric posture. However, the amputee compensated the loss of weight by shifting the upper trunk to the side of the amputation in order to get the center of gravity over the legs. Maintaining the postural stability in these two conditions of unilateral weight gain in lymphedema and unilateral weight loss in upper extremity amputation may have different mechanisms. While an individual with upper extremity amputation displaces the CoG to the amputated side to compensate the asymmetrical posture, an individual with unilateral upper extremity lymphedema probably leans toward the ipsilateral side to provide space for the larger arm. However, the lack of published data is a major challenge to make this statement in the present study.

As a variable of the LOS test, DCL quantifies how well the individual keeps the CoG in a straight line during voluntary movement from the central position to the pre-determined target. Any detected extraneous movement that disrupts the CoG from a straight line of trajectory indicates increased postural sway during the voluntary movement towards the given direction. It has been reported that subjects who have increased postural sway also have lower ability to maintain postural stability [22]. In the present study, DCL in forward, back, ipsilateral and contralateral directions was found to be significantly lower in the lymphedema group. For subjects in the lymphedema group, the average position of the CoG was in the first quadrant (Fig. 3) and already in an advanced position at the beginning of the movement toward ipsilateral and forward directions during the LOS test. Therefore DCL could not reach $100 \%$ LOS even if no extraneous movement existed. However, extraneous movements always exist during standing and voluntary movement of the CoG from the central position to the pre-determined target has been hypothesized [23]. This probably resulted in lower DCL in ipsilateral and forward directions in the lymphedema group. On the other hand, longer distance between the displaced CoG and the target on the contralateral side might also cause more extraneous movement, and probably results in lower DCL in the contralateral direction. DCL in the backward direction was also found to be low in the lymphedema group. It might be assumed that, when an individual pays full attention to lean backward to the pre-determined target, minimizing the extraneous movement in the mediolateral direction becomes difficult due to asymmetrical posture. Similar results were found in previous studies reporting that if one direction is focused for reducing postural sway, increased postural sway occurs in other directions $[23,24]$. When a subject leans backward with coordinated contraction of the anterior and posterior muscles at the ankle, it might be difficult to coordinate the contraction of the mediolateral muscles to stabilize the ankle because of the complexity of the task, as was observed in previous studies [23].

In conclusion, reduced DCL and increased postural sway, and CoG displacement to the lymphedema side, were detected in the lymphedema group. Lack of quantification of the additional weight gained in the left upper extremity due to lymphedema and the small number of participants were the main limitations of the current study. However, the differences between the two groups remain remarkable, and further studies are warranted to confirm the changes of postural stability in patients with unilateral upper extremity lymphedema. The rehabilitation team may also take the results into account for improvement of the postural stability within the overall treatment plan in breast cancer survivors.

The authors declare no conflict of interest.

\section{References}

1. Ruhe A, Fejer R, Walker B. Center of pressure excursion as a measure of balance performance in patients with non-specific low back pain compared to healthy controls: a systematic review of the literature. Eur Spine J 2011; 20: 358-68.

2. Greitemann B, Guth V, Baumgartner R. Asymmetry of posture and truncal musculature following unilateral arm amputation - a clinical, electromyographic, posture analytical and photogrammetric study. Z Orthop Ihre Grenzgeb 1996; 134: 498-510.

3. Zhang XA, Ye M, Wang CT. Effect of unilateral load carriage on postures and gait symmetry in ground reaction force during walking. Comput Methods Biomech Biomed Engin 2010; 13: 339-44.

4. Bertels T, Schmalz T, Ludwigs E. Biomechanical influences of shoulder disarticulation prosthesis during standing and level walking. Prosthet Orthot Int 2012; 36: 165-72.

5. Ahmed RL, Thomas W, Yee D, Schmitz KH. Randomized controlled trial of weight training and lymphedema in breast cancer survivors. J Clin Oncol 2006; 24: 2765-72.

6. Hoe AL, Iven D, Royle GT, Taylor I. Incidence of arm swelling following axillary clearance for breast-cancer. Br J Surg 1992; 79: 261-2.

7. Didem K, Ufuk YS, Serdar S, Zumre A. The comparison of two different physiotherapy methods in treatment of lymphedema after breast surgery. Breast Cancer Res Treat 2005; 93: 49-54.

8. Ochałek K. Prevention of lymphoedema. Contemp Oncol (Pozn) 2011; 15: 354-6.

9. Bani HA, Fasching PA, Lux MM et al. Lymphedema in breast cancer survivors: Assessment and information provision in a specialized breast unit. Patient Educ Couns 2007; 66: 311-8.

10. Brorson H, Ohlin K, Olsson G, Nilsson M. Adipose tissue dominates chronic arm lymphedema following breast cancer: an analysis using volume rendered CT images. Lymphat Res Biol 2006; 4: 199-210.

11. Balzarini A, Lualdi P, Lucarini C, Ferla S, Galli M, Crivellini M, DeConno F. Biomechanical evaluation of scapular girdle in patients with chronic arm lymphedema. Lymphology 2006; 39: 132-40.

12. Norman SA, Miller LT, Erikson HB, Norman MF, McCorkle R. Development and validation of a telephone questionnaire to characterize lymphedema in women treated for breast cancer. Phys Ther 2001; 81: 1192-205. 
13. Ko DSC, Lerner R, Klose G, Cosimi AB. Effective treatment of lymphedema of the extremities. Arch Surg 1998; 133: 452-57.

14. Bourelle S, Berge B, Gautheron V, Cottalorda J. Computerized static posturographic assessment after treatment of equinus deformity in children with cerebral palsy. J Pediatr Orthop B 2010; 19: 211-20.

15. Bakirhan S, Angin S, Karatosun V, Unver B, Günal I. A comparison of static and dynamic balance in patients with unilateral and bilateral total knee arthroplasty. Eklem Hastalik Cerrahisi 2009; 20: 93-101.

16. Gordon LG, Battistutta D, Scuffham P, Tweeddale M, Newman B. The impact of rehabilitation support services on health-related quality of life for women with breast cancer. Breast Cancer Res. Treat. 2005; 93: 217-26.

17. Vignes S, Porcher R, Maria A, Dupuy A. Long-term management of breast cancer-related lymphedema after intensive decongestive physiotherapy. Breast Cancer Res Treat 2007; 101: 285-90.

18. Matsuo T, Hashimoto M, Koyanagi M, Hashizume K. Asymmetric load-carrying in young and elderly women: relationship with lower limb coordination. Gait Posture 2008; 28: 517-20.

19. Haddad JM, Rietdyk S, Ryu JH, Seaman JM, Silver TA, Kalish JA, Hughes CM. Postural asymmetries in response to holding evenly and unevenly distributed loads during self-selected stance. J Motor Behav 2011; 43: 345-55.

20. Blaszczyk JW, Prince F, Raiche M, Hebert R. Effect of ageing and vision on limb load asymmetry during quiet stance. J Biomech 2000; 33: 1243-8.

21. Genthon N, Rougier P. Influence of an asymmetrical body weight distribution on the control of undisturbed upright stance. J Biomech 2005; 38: 2037-49.

22. Liaw MY, Chen CL, Pei YC et al. Comparison of the static and dynamic balance performance in young, middle-aged, and elderly healthy people. Chang Gung Med J 2009; 32: 297-304.

23. Balasubramaniam R, Turvey MT. The handedness of postural fluctuations. Hum Movement Sci 2000; 19: 667-84.

24. Balasubramaniam R, Riley MA, Turvey MT. Specificity of postural sway to the demands of a precision task. Gait Posture 2000; 11: $12-24$.

\section{Address for correspondence}

\section{Salih Angin}

Dokuz Eylül University

School of Physical Therapy and Rehabilitation

Sağlık Kampusü, İnciraltı 35340,

Izmir, Turkey

tel. +90 (232) 4124934

fax +90 (232) 4124946

e-mail: salih.angin@deu.edu.tr

Submitted: 10.04 .2013

Accepted: 17.12 .2013 